i-DUST 2010, $03002(2011)$

DOI: 10.1051 idust/201103002

(C) Owned by the authors, published by EDP Sciences, 2011

\title{
Analysis of scalp EEG recorded in a low-noise environment
}

\author{
A. Shahidi Zandi ${ }^{1}$, G. Dumont ${ }^{1, a}$, M. Yedlin ${ }^{1}$, Ph. Lapeyrie ${ }^{3}$, C. Sudre ${ }^{2}$, \\ and S. Gaffet ${ }^{2}$ \\ ${ }^{1}$ Department of Electrical \& Computer Engineering, University of British Columbia \\ Vancouver, BC, V6T 1Z4, Canada \\ 2 Laboratoire Souterrain à Bas Bruit de Rustrel, Observatoire de la Côte d'Azur, \\ 84400 Rustrel, France \\ ${ }^{3}$ Hôpital Universitaire Carémeau, Place du Pr. Robert Debré, 30029 Nîmes Cedex 9, France
}

\begin{abstract}
This study investigates the effects of a low-noise environment when used for recording the scalp electroencephalogram (EEG). Analysis of the EEG recordings from three volunteers confirms that clean EEG signals can be acquired in the LSBB shielded capsule without the need for notch filtering. Also, using different setups for acquiring EEG, statistical analysis reveals that the laptop computer and the patient module do not introduce any noise on the recorded signals. Moreover, the current study shows that the counting task as a mental activity can be better detected using the EEG acquired in the capsule since the relative energy of the beta band is significantly higher in this environment. Those results demonstrate the potential of the LSBB capsule for novel EEG studies.
\end{abstract}

\section{INTRODUCTION}

Electroencephalography which involves the recording and analysis of electric signals generated by the brain has long been used to diagnose various brain dysfunctions and more recently to monitor anesthesia in the operating room and sedation in the intensive care unit. For instance, the electroencephalogram (EEG) continues to play a major role in the diagnosis and management of epilepsy [1,2].

The EEG is generally described in terms of activity in different frequency bands: the delta band $(0-4 \mathrm{~Hz})$, the theta band $(4-7 \mathrm{~Hz})$, the alpha band $(8-12 \mathrm{~Hz})$, the beta band $(12-30 \mathrm{~Hz})$, and the gamma band $(30-100 \mathrm{~Hz})$. In the last decade, intra-operative EEG monitoring has become increasingly used to assess the depth of hypnosis of patients in response to hypnotic agents such as propofol.

A particular such monitor is the NeuroSense ${ }^{\mathrm{TM}}$, based on wavelet analysis primarily in the gammaband, see [3] for details of the algorithm. Although this is still subject to debate, the gamma-band has recently been associated with the somatosensory cortex, with a decrease in gamma band activity linked to cognitive decline, and an increased theta/gamma ratio associated with memory impairment [4]. Because power line interference will result in significant $50 \mathrm{or} 60 \mathrm{~Hz}$ corruption of the EEG right in the gamma band, any studies of the gamma band must be performed on an EEG that was first processed though a 50 or $60 \mathrm{~Hz}$ notch filter. While effective at removing the resulting electromagnetic (EM) interference, such a notch filter is also bound to remove some valuable information about the gamma band.

In this pilot study, our objective was to establish whether by acquiring EEG recordings in the LSBB $^{\mathrm{b}}$ shielded capsule we could do away with notch filters. Secondary objectives were to assess

\footnotetext{
a e-mail: guyd@ece.ubc.ca

b Laboratoire Souterrain à Bas Bruit de Rustrel

This is an Open Access article distributed under the terms of the Creative Commons Attribution-Noncommercial License 3.0, which permits unrestricted use, distribution, and reproduction in any noncommercial medium, provided the original work is properly cited.
} 


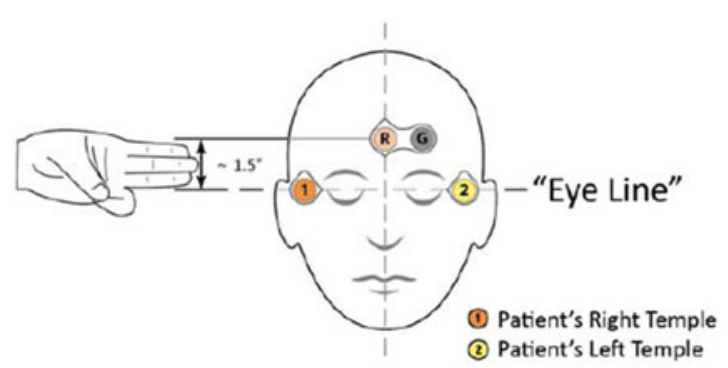

Figure 1. The montage used to acquire the EEG signals. The electrode " $R$ " is used as reference while the electrode "G" is the ground.

i) the interference from the laptop computer and the NeuroSense patient module and ii) the effect of the capsule on recognition of some mental activities using EEG.

\section{EEG DATA}

A NeuroSense $\mathrm{e}^{\mathrm{TM}}$ patient module PM-701 connected to a laptop running on battery power was used to acquire the scalp EEG of 3 volunteers sitting in a reclined position. The PM-701 is a two-channel (4 electrodes) EEG recording system for bilateral monitoring, has a bandwidth of $0.125-300 \mathrm{~Hz}$ with the noise less than $2 \mu \mathrm{Vpp}$ from 0.125 to $100 \mathrm{~Hz}$, and provides a raw EEG sampled at $900 \mathrm{~Hz}$. No external power source was required by the system. The $900 \mathrm{~Hz}$ sampled raw, unfiltered (all filters on the PM701 were turned off) EEG data were acquired using the NeuroSense NS-701 software running under Windows Vista on a laptop running under battery power and connected to the PM-701 via a USB cable DC-701. The PM-701 is powered by the laptop via the USB cable. The resulting data were then analyzed using MATLAB. The EasyPrep ${ }^{\mathrm{TM}}$ EK-701 electrodes were used to acquire bilateral EEG as depicted on Figure 1.

For comparison purposes, the EEG signals were first recorded in a hospital environment, namely the orthodontal surgery suite at the Carémeau University Hospital in Nîmes. EEG was then acquired on the same three subjects in the $1500 \mathrm{~m}^{3}$ shielded capsule at the LSBB. Three setups were studied: 1) subject, PM-701 and laptop in capsule, 2) subject, and PM-701 in capsule, laptop outside, 3) subject in capsule, PM-701 and laptop outside. In each setup, the subjects were asked to relax or count backward in 7 under three different conditions: $24 \mathrm{~V}$ lights on, $24 \mathrm{~V}$ lights off, and total power blackout, i.e. neither power nor ventilation in that section of the tunnel.

\section{DATA ANALYSIS}

To analyze the effects of the shielded capsule, EEG recordings of 3 subjects in the two environments (i.e., hospital and capsule) were studied. Figure 2(a) presents a ten-second raw EEG segment of the right channel recorded in the hospital from Subject 1, overlayed by the notch-filtered EEG, when the subject is relaxed and lights are off. Figure 2(b) shows a ten-second EEG interval from the right channel of the same subject, while the subject is relaxed in the capsule with blackout condition. As can be seen, whereas the raw EEG recorded in the hospital is highly corrupted by the $50 \mathrm{~Hz}$ noise, the power line interference is not seen in the EEG recorded in the capsule.

\subsection{Power spectral analysis}

Studying the power spectra of the EEG recordings from both environments clearly shows the advantage of the LSBB shielded capsule in acquiring clean EEG signals. 
A. Shahidi Zandi et al.: Analysis of scalp EEG recorded in a low-noise environment



(a)

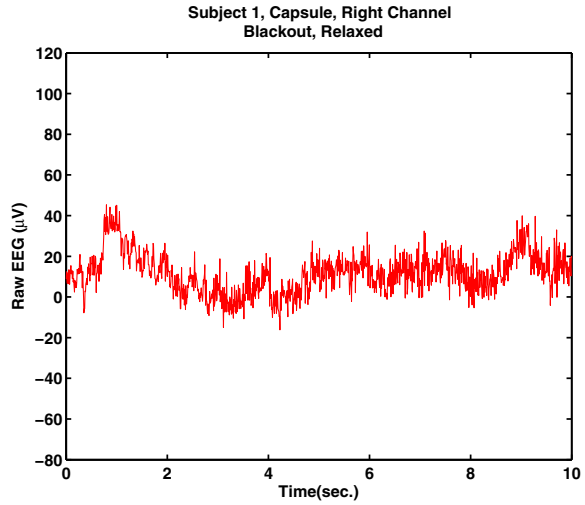

(b)

Figure 2. Ten-second EEG segments of the right channel from Subject 1, while relaxed: a) Hospital, lights off; b) Capsule, blackout.

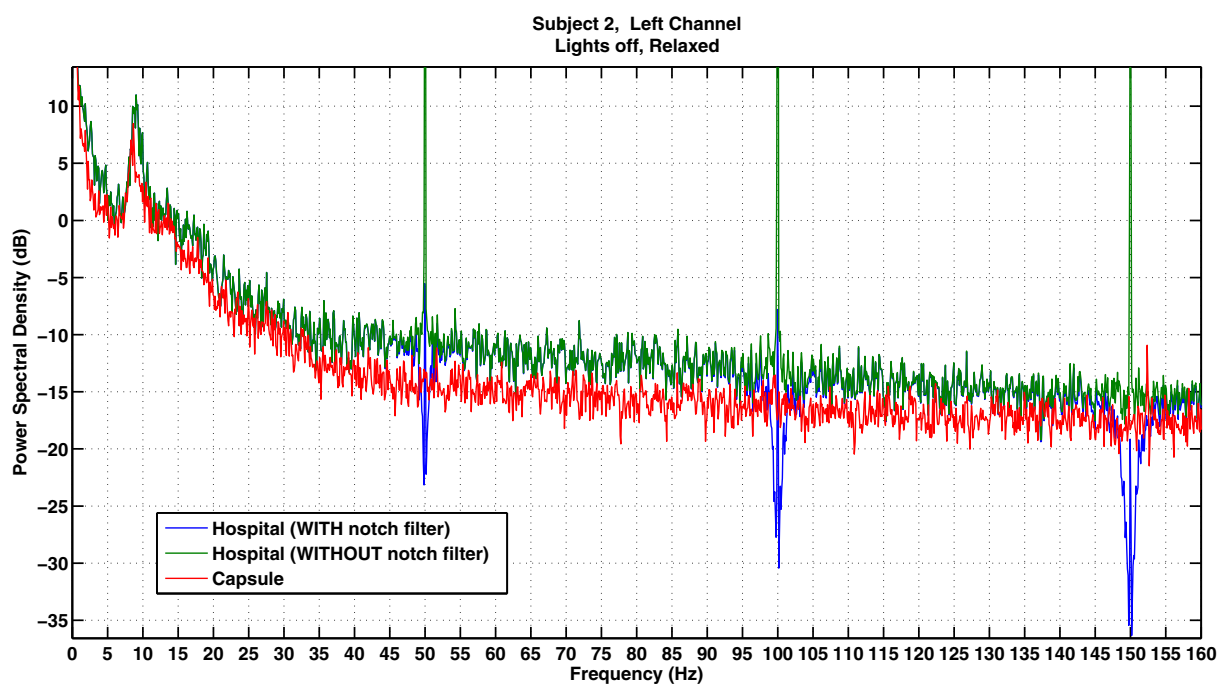

Figure 3. The power spectra of the the left channel EEG for Subject 2, no-light/relaxed: i) in the hospital with and without notch filtering and ii) in the capsule.

Figure 3 displays power spectral densities (PSDs) of the left channel EEG from Subject 2 for the no-light/relaxed situation: i) acquired in the surgical suite at the hospital with and without notch filter and ii) acquired in the capsule. In the hospital, the presence of the $50 \mathrm{~Hz}$ power line interference and its harmonics at 100 and $150 \mathrm{~Hz}$ are striking. Clearly, for assessment of the gamma band activity, notch filtering is required. However, as the results of notch filtering show, the filtered signal is distorted with loss of information. On the other hand, an EEG acquired in the capsule clearly shows that there is no EM interference.

Investigating the interference from the laptop and NeuroSense patient module, the spectral densities from the three aforementioned setups for acquiring EEG in the capsule (i.e. module and laptop in capsule, laptop outside, and laptop and module outside) were statistically compared. For this purpose, a geodesic distance recently proposed by Georgiou [5] was employed. Given two PSD functions $P_{1}(\theta)$ and $P_{2}(\theta)(\theta \in[-\pi, \pi])$, the geodesic distance $d_{g}\left(P_{1}, P_{2}\right)$, providing a pseudo-metric on the cone of 
i-DUST 2010

Table 1. Results of the one-sided t-test ( $p$-values), comparing the PSDs of different setups for the three subjects. Setup 1: subject, PM-701 and laptop in capsule; Setup 2: subject, and PM-701 in capsule, laptop outside; Setup 3: subject in capsule, PM-701 and laptop outside.

\begin{tabular}{|c|c|ccc|}
\hline \multirow{3}{*}{ Subject } & & \multicolumn{3}{|c|}{ Setups Compared } \\
\cline { 3 - 5 } & Channel & $\mathbf{1 - 2}$ & $\mathbf{1 - 3}$ & $\mathbf{2 - 3}$ \\
\hline \hline 1 & Right & 0.2117 & 0.5621 & 0.8589 \\
& Left & 0.2490 & 0.3775 & 0.8210 \\
\hline 2 & Right & 0.0592 & 0.1281 & 0.6126 \\
& Left & 0.0093 & 0.0933 & 0.0668 \\
\hline 3 & Right & 0.3718 & 0.8433 & 0.0340 \\
& Left & 0.2270 & 0.3045 & 0.2909 \\
\hline
\end{tabular}

PSD functions, is defined as

$$
d_{g}\left(P_{1}, P_{2}\right)=\sqrt{\int_{-\pi}^{\pi}\left(\log \frac{P_{1}(\theta)}{P_{2}(\theta)}\right)^{2} \frac{d \theta}{2 \pi}-\left(\int_{-\pi}^{\pi} \log \frac{P_{1}(\theta)}{P_{2}(\theta)} \frac{d \theta}{2 \pi}\right)^{2}} .
$$

Analyzing the EEG acquired using different setups, each EEG recording was segmented into nonoverlapping epochs with the length of $30 \mathrm{~s}$, and the corresponding PSD was obtained. Then, the distance between the PSDs of each two epochs was calculated using (1). For each setup, this resulted in a set of numbers, showing the PSD intra-distance values of that setup. In the next step, choosing every two setups, the distances between PSDs of epochs in one setup and those of the other were computed, providing three sets of the PSD inter-distance numbers (i.e., Setups 1-2, Setups 1-3, and Setups 2-3).

To compare the PSDs of each two setups, one setup was selected as the reference, and the reference intra-distance set was statistically compared to the set of the PSD distances between the two setups (inter-distance) using a one-sided t-test with the significance level of 5\% and the alternative hypothesis that the mean of geodesic distance between two setups is greater than the mean of intra-distance values of the reference (i.e., geodesic distance between PSDs of different epochs of the reference signal). In fact, the alternative represents the case that the power spectrum of a setup is significantly different from that of the reference, since their average distance is greater than the average PSD distance within the reference.

Table 1 presents the $p$-values of the t-test, when comparing different setups for all subjects. According to the results, only two cases reject the null hypothesis (i.e., $p<0.05$ ): Subject 2 , Setups 1-2, left channel and Subject 3, Setups 2-3, right channel. However, the null hypothesis cannot be rejected in most cases, showing that PSDs of three different setups are not statistically different. This indicates that neither laptop nor patient module PM-701 interfered with the acquired EEG data.

\subsection{Time-frequency analysis}

In order to evaluate the influence of the shielded capsule on recognition of the mental activities (here, counting), the Stockwell transform [6], also known as the $S$ transform, was employed to quantify the frequency content of EEG signals versus time (i.e., EEG time-frequency representation). The Stockwell transform provides frequency-dependent resolution and maintains a direct relationship with the Fourier transform of the given signal, allowing to detect high-frequency bursts (i.e., high time resolution at high frequencies) and to have good frequency resolution on long period signals. Given signal $x(t)$, the Stockwell transform of $x(t)$ is defined as

$$
S(\tau, f)=\int_{-\infty}^{\infty} x(t) \frac{|f|}{\sqrt{2 \pi}} e^{-\frac{(\tau-t)^{2} f^{2}}{2}} e^{-j 2 \pi f t} d t
$$


A. Shahidi Zandi et al.: Analysis of scalp EEG recorded in a low-noise environment

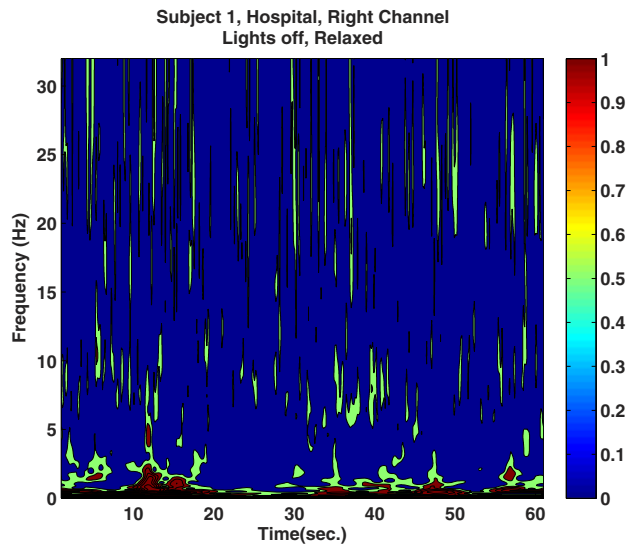

(a)

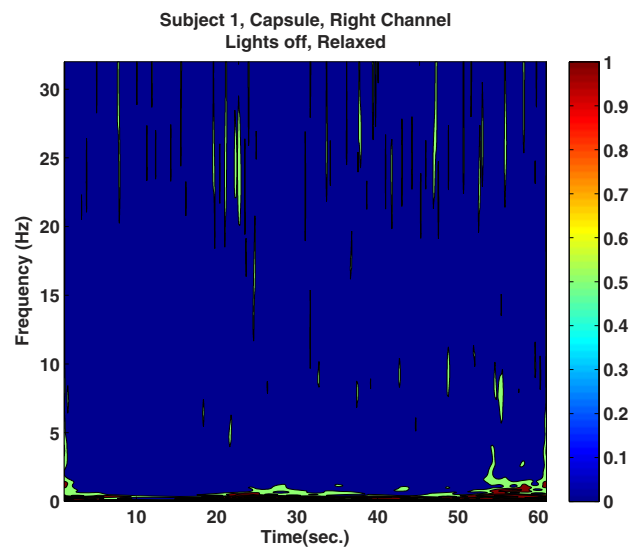

(c)

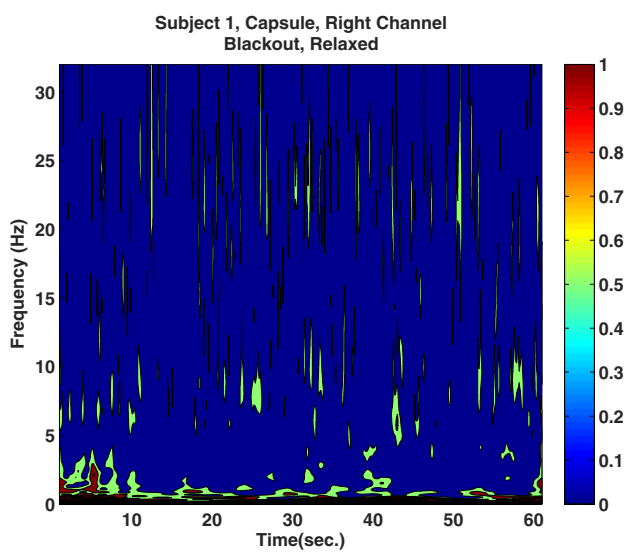

(e)



(b)

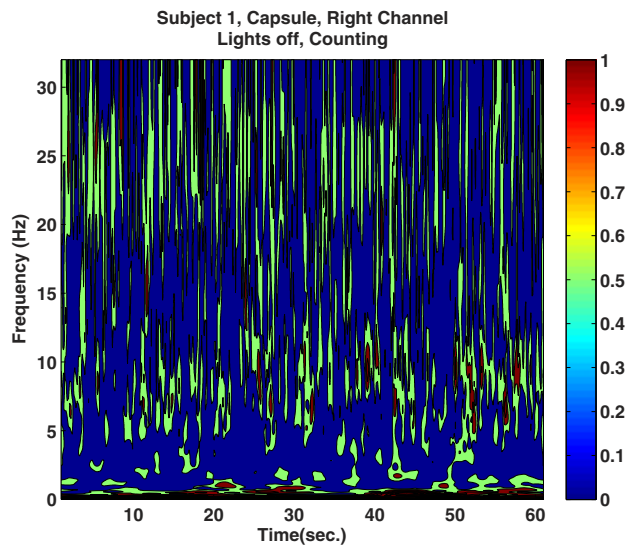

(d)

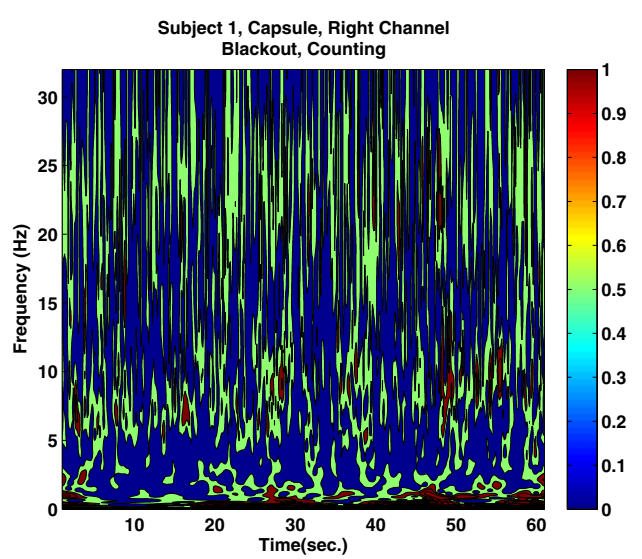

(f)

Figure 4. The normalized magnitude of the Stockwell transform of sixty-second EEG segments of the right channel from Subject 1, acquired in different situations: a) Hospital, lights off, relaxed; b) Hospital, lights off, counting; c) Capsule, lights off, relaxed; d) Capsule, lights off, counting; e) Capsule, blackout, relaxed; f) Capsule, blackout, counting. 


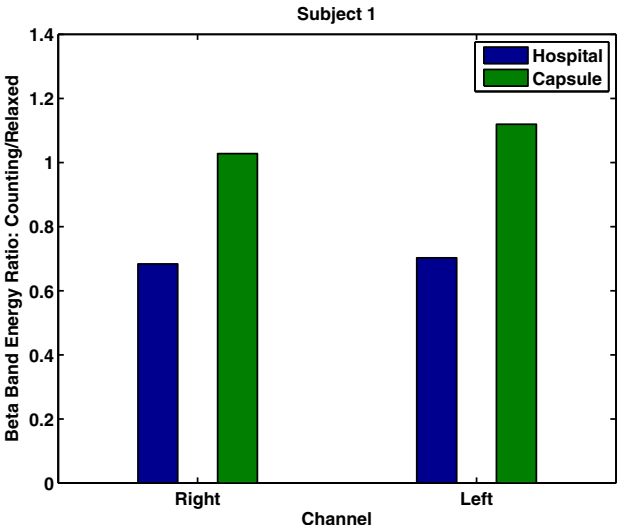

(a)

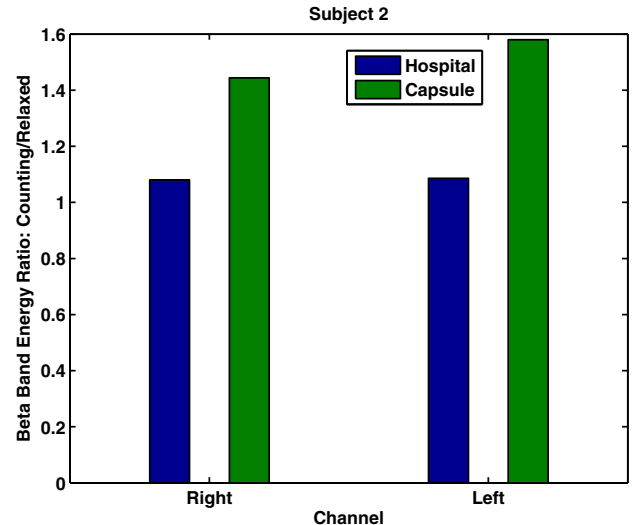

(b)



(c)

Figure 5. The ratio of the beta band energy in counting state to that in the relaxed state in both environments: a) Subject 1; b) Subject 2; c) Subject 3 .

Figure 4 shows the normalized magnitude of the Stockwell transform of EEG segments with the length of $60 \mathrm{~s}$ from Subject 1 (right channel), recorded in different situations. Figures 4(a) and 4(b) present this magnitude for the recordings of no-light/relaxed and no-light/counting situations in the hospital. The results of the capsule for no-light/relaxed, no-light/counting, blackout/relaxed, and blackout/counting situations are seen in Figures 4(c)-4(f). As the results show, there is no significant difference between the relaxed and counting states in the hospital. In the capsule, however, a noticeable energy increase can be seen in the beta band for both the no-light and blackout situations as the mental activity occurs.

To quantitatively evaluate the effect of the capsule on detecting the counting task using EEG, the ratio of the EEG energy in beta band in the counting state to that in the relaxed state was calculated using the Stockwell transform for all subjects. For this purpose, each EEG recording was segmented into non-overlapping epochs with the length of $30 \mathrm{~s}$. Then, the energy of the beta band $(12-30 \mathrm{~Hz})$ was computed by summing the squared values of the Stockwell transform magnitude in the corresponding region of the time-frequency space for each epoch. In the next step, the total energy of the beta band for each situation (i.e., relaxed or counting) was obtained by summing the energies calculated for the corresponding epochs. Figure 5 shows the ratio of the beta band energy in the counting state to that in the relaxed state for the three subjects in both environments. In each case, the energy ratio is shown for 
A. Shahidi Zandi et al.: Analysis of scalp EEG recorded in a low-noise environment

both the right and left channels. According to the results, for all subjects the energy ratio in the capsule is significantly higher than the energy ratio in the hospital, revealing that the mental activity can be recognized better from the EEG acquired in the LSBB shielded capsule.

\section{DISCUSSION}

The purpose of this study was to investigate the effects of the LSBB shielded capsule, when used as an environment for recording the scalp EEG signals.

Our preliminary findings confirm the hypothesis that EEG signals acquired in the capsule do not require notch filtering. In addition, the statistical analysis of the power spectra of the EEG acquired using different setups in the capsule shows that neither patient module nor the laptop introduces any noise on the acquired EEG signals. Comparing the EEG recorded in the hospital and capsule, this study also reveals that the counting task as a mental activity is better recognizable using the EEG recorded in the capsule. The results show that the ratio of the EEG beta band energy during the counting task to that of the relaxed state is significantly higher in the capsule.

Because it was put together fairly rapidly, this study has some limitations. First, due to the temperature in the capsule, subjects sweated profusively during the experiment, creating artifacts on the signals. Also, the subjects were not sitting very comfortably and thus were not completely relaxed. The other limitation of this work is that the counting task may not have been sufficient to clearly detect beta waves and the subjects were not told whether to open or close their eyes while counting. Finally, a subject briefly dozed off during the test.

\section{CONCLUSIONS}

This first pilot study does confirm the potential of the capsule for novel EEG data studies taking advantage of this unique low-noise environment to detect physiologically-based minute EEG patterns normally buried in noise.

More informative studies need to be designed to address the limitations of the current work and to further assess the advantages of acquiring EEG signals in the shielded capsule. Also, one might consider using a research-grade rather than a clinical grade EEG system capable of faster sampling as indeed fast brain oscillations may be of interest $[7,8]$.

The authors would like to thank all the providers of funding and support for this project. LSBB and the région PACA provided the infrastructure. Funding was also provided by the Natural Sciences and Engineering Research Council of Canada (NSERC).

\section{References}

[1] M.E. Saab, J. Gotman, Clinical Neurophysiology 116(2), 427 (2005)

[2] A. Shahidi Zandi, M. Javidan, G.A. Dumont, R. Tafreshi, IEEE Transactions on Biomedical Engineering 57(7), 1639 (2010)

[3] T. Zikov, S. Bibian, G.A. Dumont, M. Huzmezan, C.R. Ries, IEEE Transactions on Biomedical Engineering 53(4), 617 (2006)

[4] D. Moretti, C. Fracassi, M. Pievani, C. Geroldi, G. Binetti, O. Zanetti, K. Sosta, P. Rossini, G. Frisoni, Clinical Neurophysiology 120(2), 295 (2009)

[5] T. Georgiou, IEEE Signal Processing Letters 14(8), 561 (2007)

[6] R.G. Stockwell, L. Mansinha, R.P. Lowe, IEEE Transactions on Signal Processing 44(4), 998 (1996) 


\section{i-DUST 2010}

[7] H. Scheer, G. Curio, M. Burghoff, Measurement of high frequency EEG signals with very low noise amplifiers: Brain oscillations above $600 \mathrm{~Hz}$ measured non-invasively, in IFMBE Proceedings (2008), Vol. 22, pp. 1302-1304

[8] R. Zelmann, F. Mari, J. Jacobs, M. Zijlmans, R. Chander, J. Gotman, Automatic detector of high frequency oscillations for human recordings with macroelectrodes, in 32nd Annual International Conference of the IEEE EMBS Proceedings (2010), pp. 2329-2333 